\title{
LIMIT FUNCTIONS OF ITERATES OF ENTIRE FUNCTIONS ON PARTS OF THE JULIA SET
}

\author{
PETER BEISE AND JÜRGEN MÜLLER \\ (Communicated by Nimish Shah)
}

\begin{abstract}
We show that the iterates of entire functions have maximal sets of limit functions on many small subsets of the corresponding Julia set.
\end{abstract}

\section{IntroduCtion AND RESUlts}

Let $f$ be an entire function. We always assume that $f$ is not a polynomial of degree 0 or 1 . The Julia set $J=J(f)$ of $f$ is defined as the set of all $z \in \mathbb{C}$ so that the iterates $f^{\text {on }}$ do not form a normal family in any neighborhood of $z$. For properties of the Julia set we refer to the expository articles [2] and [10].

For $z \in J$, let $\omega(z) \subset J$ denote the set of limit points of $\left(f^{\circ n}\right)$. Then $\omega(z)$ is nonempty if and only if $z$ belongs to $J \backslash I$, where $I=I(f)$ is the escaping set of $f$ (that is, the set of all $z \in \mathbb{C}$ such that $f^{\circ n}(z)$ tends to $\infty$ ). In particular, for polynomials we have $\omega(z) \neq \emptyset$ for all $z \in J$. Using the terminology that a property is said to be satisfied by quasi all elements in a Baire space if it is satisfied on a residual set in this space, it is known that $\omega(z)=J$ for quasi all $z \in J$. A proof is found e.g. in 1 .

Our aim is to replace limit points by limit functions on subsets of $J$. For an arbitrary set $L \subset J$ let $\omega(L)=\omega(L, f)$ be the set of all functions $g: L \rightarrow J$ so that $f^{\circ n_{j}} \rightarrow g$ pointwise on $L$ for some subsequence $\left(f^{\circ n_{j}}\right)$ of $\left(f^{\circ n}\right)$. We first note some obvious facts. Firstly, only functions that are pointwise limits of continuous functions on $L$ with values in $J$ can belong to $\omega(L)$. By $B(L, J)$ we denote the set of those functions. In particular, $B(L):=B(L, \mathbb{C}$ ) is the Baire class 1 (see [5. Theorem (24.10) and Exercise (24.13)]). Secondly, if $g \in \omega(L)$, then we have $f^{\circ m} \circ g \in \omega(L)$ for all nonnegative integers $m$.

The first result shows that limit functions can only exist on sets which are topologically small:

Proposition 1. If $L$ is of second category in $J$, then $\omega(L)=\emptyset$.

The proof of the proposition will show also that $g \equiv \infty$ cannot appear as a limit function on a set of second category. On the other hand, $g \equiv \infty$ may be a limit function on sets of full planar Lebesgue measure, as for instance in the case of postcritically finite maps in the cosine family, where actually the escaping set has full measure in the sense that the complement has measure zero (see, e.g., [10, p. 324]).

Received by the editors January 25, 2012.

2010 Mathematics Subject Classification. Primary 37F99, 30D05, 30K20.

Key words and phrases. Julia set, iteration of entire functions, universal functions. 
For a compact set $E$ and a closed set $M$ in $\mathbb{C}$ the set of continuous functions from $E$ to $M$ is denoted by $C(E, M)$. We put $\|f\|_{E}:=\sup _{z \in E}|f(z)|$ and endow $C(E, M)$ with the complete metric $d(f, g):=\|f-g\|_{E}$. Moreover, we define $\omega_{u}(E):=$ $\omega_{u}(E, f)$ to be the limit set of $\left.f^{\circ n}\right|_{E}$ in $C(E, J)$. Then, obviously, $\omega_{u}(E) \subset \omega(E)$ and $\omega_{u}(E) \subset C(E, J)$. Our main result will show that for 'many' compact sets $E$ the limit set $\omega_{u}(E)$ equals $C(E, J)$.

In order to give the precise formulation, we recall the following. Given some metric space $(X, d)$ and compact, nonempty $E, F \subset X$, the Hausdorff distance of $E$ and $F$ is defined as

$$
d_{H}(E, F):=\max \{\delta(E, F), \delta(F, E)\},
$$

where

$$
\delta(E, F):=\max _{x \in E} \operatorname{dist}(x, F) .
$$

Let $\mathcal{K}(X):=\{E \subset X: E$ nonempty and compact $\}$. If $d$ is a complete metric, then $\left(\mathcal{K}(X), d_{H}\right)$ is known to be a complete metric space. Morover, if $(X, d)$ is separable, so is $\left(\mathcal{K}(X), d_{H}\right)$.

A closed subset $E$ of the unit circle $\mathbb{T}$ is called a Kronecker set if the set $\{z \mapsto$ $\left.\left.z^{m}\right|_{E}: m \in \mathbb{N}\right\}$ is dense in $C(E, \mathbb{T})$. In [6], Körner shows that quasi all sets in $\mathcal{K}(\mathbb{T})$ are Kronecker sets. It is easily seen that for every integer $d \geq 2$ the reasoning in 6 ] also yields that $\omega_{u}\left(E, z \mapsto z^{d}\right)=C(E, \mathbb{T})$ for quasi all $E \in \mathcal{K}(\mathbb{T})$. Inspired by this observation, we show that this property extends to arbitrary entire functions (not being a polynomial of degree 0 or 1 ):

Theorem 1. For quasi all sets $E \in \mathcal{K}(J)$ we have $\omega_{u}(E)=C(E, J)$.

Remark 1. For an arbitrary entire function $f$ the Julia set $J=J(f)$ is known to be perfect. It can be shown that for a complete metric space $(X, d)$ which is separable and perfect, quasi all $E \in \mathcal{K}(X)$ are perfect (cf. [5, p. 42]). Since finite (and even countable) intersections of dense $G_{\delta}$-sets are dense $G_{\delta}$, quasi all sets as in Theorem 1 are perfect and thus in particular uncountable.

Remark 2. If $L$ is the union of an increasing sequence of compact sets $E_{k}$ with $\omega_{u}\left(E_{k}\right)=C\left(E_{k}, J\right)$, then $\omega(L)=B(L, J)$.

(Indeed: Let $g \in B(L, J)$. Then there is a sequence of continuous functions $g_{k}: L \rightarrow J$ with $g_{k} \rightarrow g$ pointwise on $L$. Moreover, to each $k$ there exists some $n_{k}$ with

$$
\left\|g_{k}-f^{\circ n_{k}}\right\|_{E_{k}}<1 / k .
$$

Then $f^{\circ n_{k}} \rightarrow g$ pointwise on $L$.)

In particular, from Theorem [1 it follows that $\omega(E)=B(E, J)$ for quasi all $E \in \mathcal{K}(J)$.

If $L$ is a set with $\omega(L)=B(L, J)$, then in particular $\omega(z)=J$ for all $z \in L$. Thus, such sets $L$ cannot contain periodic points of $f$. It is well-known that the set of repelling periodic points is dense in $J$.

Remark 3. If $f$ is an entire function with $J(f)=\mathbb{C}$, then $\omega_{u}(E)=C(E):=C(E, \mathbb{C})$ and $\omega(E)=B(E)$ for quasi all $E \in \mathcal{K}(\mathbb{C})$. Each entire function such that the singular values are contained in a bounded set in $\mathbb{C}$ and are strictly preperiodic or escape to $\infty$ have maximal Julia set $J(f)=\mathbb{C}$ (see e.g. [10, Corollary 3.14]). 
In particular, this holds for the exponential function exp. Thus, we have $\omega_{u}(E, \exp )=C(E)$ and $\omega(E, \exp )=B(E)$ for quasi all compact sets $E$ in $\mathbb{C}$.

On the other hand, the union of all sets $L$ with $\omega(L$, exp $)=B(L)$ lies in a set of vanishing (planar Lebesgue) measure, which follows from the fact that the pointwise limit set $\omega(z, \exp )$ equals the orbit $\left\{\exp ^{\circ m}(0): m \in \mathbb{N}_{0}\right\}$ of the asymptotic value 0 for almost all $z \in \mathbb{C}$ (see [7]; cf. also [9]). It would be interesting to know if $\omega(L, \exp ) \neq \emptyset$ for some set of positive measure.

In the case of postcritically finite cosine maps $f$ (where also $J(f)=\mathbb{C}$ ), the sets $L$ with $\omega(L, f)=B(L)$ consist of landing points of dynamic rays (see [10, Theorem 5.4]).

\section{Proofs}

Proof of Proposition 1, As remarked above, $\omega(z)=J$ for quasi all $z \in J$. It turns out that virtually the same proof shows that, for each infinite subset $\Lambda$ of the nonnegative integers $\mathbb{N}_{0}$, the set $\omega(z, \Lambda)=\omega(z, f, \Lambda)$ of limit points of $\left(f^{\circ n}\right)_{n \in \Lambda}$ equals $J$ for quasi all $z \in J$. For sake of completeness we sketch the proof:

According to the Universality Criterion (see, e.g., 4]) it suffices to show that for each pair of nonempty open sets $U, V$ in $J$ there is some $n \in \Lambda$ so that $f^{\circ n}(U) \cap V \neq \emptyset$ (note that, since $J$ does not contain isolated points, $\omega(z, \Lambda)=\mathbb{C}$ if $\left\{f^{\circ n}: n \in \Lambda\right\}$ is dense in $\mathbb{C}$ ).

Suppose that $f^{\circ n}(U) \cap V=\emptyset$ for all $n \in \Lambda$. Since $U$ is relatively open in $J$, there exists an open set $U_{0}$ in $\mathbb{C}$ with $U_{0} \cap J=U$. From the invariance of $J$ under $f$ we obtain $f^{\circ n}\left(U_{0}\right) \cap V=\emptyset$ for all $n \in \Lambda$. Since $J$ has no isolated points, Montel's Theorem (see, e.g., [3, Theorem 3.2]) implies that $\left(\left.f^{\circ n}\right|_{U_{0}}\right)_{n \in \Lambda}$ is a normal family. This contradicts the fact that no subsequence of $\left(f^{\circ n}\right)_{n \in \mathbb{N}_{0}}$ is normal on any open set intersecting $J$ (cf. [11, p. 38] for the case of polynomials; the proof for transcendental entire functions runs along the same lines).

Now, suppose that $g \in \omega(L)$. Then $f^{\circ n_{j}} \rightarrow g$ pointwise on $L$ for some sequence $\left(n_{j}\right)$ in $\mathbb{N}_{0}$. Then $\omega\left(z,\left\{n_{j}: j \in \mathbb{N}\right\}\right)=\{g(z)\}$ for all $z \in L$. The above considerations show that $L$ has to be of first category.

Proof of Theorem 1. It suffices to prove that the set of iterates $f^{\circ n}$ is dense in $C(E, J)$.

We consider the space $C(\mathbb{C})$ of all (complex-valued) continuous functions on $\mathbb{C}$ with the topology of uniform convergence on compact subsets of $\mathbb{C}$. Then $C(\mathbb{C})$ is a Fréchet space and, according to the (Stone-)Weierstrass theorem, the polynomials in two real variables with coefficients having rational real and imaginary parts form a dense set in $C(\mathbb{C})$. Let $\left(p_{j}\right)_{j \in \mathbb{N}}$ be an enumeration of these polynomials.

For $j \in \mathbb{N}$ we define

$$
\mathcal{B}_{j}:=\left\{F \in \mathcal{K}(J):\left\|p_{j}-f^{\circ N}\right\|_{F}<\delta\left(p_{j}(F), J\right)+2 / j \text { for some } N \in \mathbb{N}\right\} .
$$

The continuity of $f$ and $p_{j}$ implies that $\mathcal{B}_{j}$ is open in $\mathcal{K}(J)$. We show that $\mathcal{B}_{j}$ is also dense in $\mathcal{K}(J)$.

For that purpose, let $j$ be fixed and let $E \in \mathcal{K}(J)$ and $\varepsilon>0$. We choose $0<\delta<\varepsilon$ so that

$$
\left|p_{j}(z)-p_{j}(w)\right|<1 / j \text { whenever }|z| \leq \max \{|\zeta|: \zeta \in E\} \text { and }|z-w| \leq 2 \delta .
$$


Moreover, we choose $z_{1}, \ldots, z_{m} \in E$ so that for

$$
U_{k}:=\left\{z:\left|z-z_{k}\right| \leq \delta\right\} \cap J \quad(k=1, \ldots, m)
$$

we have

$$
E \subset \bigcup_{k=1}^{m} U_{k} .
$$

If $f$ is a polynomial, $J$ is compact in $\mathbb{C}$. If $f$ is transcendental, $J$ is closed and unbounded in $\mathbb{C}$. Moreover, from Picard's theorem it follows that $f$ has at most one exceptional point in $\mathbb{C}$, that is, a point with finite backward orbit. Since the sets $p_{j}\left(U_{1}\right), \ldots, p_{j}\left(U_{m}\right)$ are compact, we can choose a compact subset $J_{0}$ of $J$ which does not contain the exceptional point (if it exists) and so that

$$
\operatorname{dist}\left(J_{0}, p_{j}\left(U_{k}\right)\right)<\operatorname{dist}\left(J, p_{j}\left(U_{k}\right)\right)+1 / j
$$

for $k=1, \ldots, m$.

From the fact that the repelling periodic points of $f$ are dense in the Julia set $J$, it follows that there is an $N \in \mathbb{N}$ with

$$
f^{\circ N}\left(U_{k}\right) \supset J_{0}
$$

for $k=1, \ldots, m$. This is well-known for the case of polynomials $f$ (see, e.g., [3. Theorem III 3.2], 8, Corollary 14.2]) and can be proved in a similar way for transcendental entire functions; cf. [2, p. 156].

Hence, for every $k=1, \ldots, m$ we can find a point $w_{k} \in U_{k}$ so that

$$
\operatorname{dist}\left(f^{\circ N}\left(w_{k}\right), p_{j}\left(U_{k}\right)\right)=\operatorname{dist}\left(J_{0}, p_{j}\left(U_{k}\right)\right)<\operatorname{dist}\left(J, p_{j}\left(U_{k}\right)\right)+1 / j .
$$

According to (1) we obtain

$$
\begin{aligned}
\left|f^{\circ N}\left(w_{k}\right)-p_{j}\left(w_{k}\right)\right| & \leq \operatorname{dist}\left(f^{\circ N}\left(w_{k}\right), p_{j}\left(U_{k}\right)\right)+1 / j \\
& <\operatorname{dist}\left(J, p_{j}\left(U_{k}\right)\right)+2 / j \leq \operatorname{dist}\left(J, p_{j}\left(w_{k}\right)\right)+2 / j
\end{aligned}
$$

for $k=1, \ldots, m$. Putting $F:=\left\{w_{1}, \ldots, w_{m}\right\}$, this shows that $F \in \mathcal{B}_{j}$. Moreover, from the definiton of $U_{k}$ we obtain

$$
d_{H}(F, E) \leq \delta<\varepsilon,
$$

and thus $\mathcal{B}_{j}$ is dense in $\mathcal{K}(J)$.

According to Baire's theorem, the set $\bigcap_{j \in \mathbb{N}} \mathcal{B}_{j}$ is a dense $G_{\delta}$-subset of $\mathcal{K}(J)$. Therefore, it suffices to show that every set $E \in \bigcap_{j \in \mathbb{N}} \mathcal{B}_{j}$ has the property that $\left\{\left.f^{n}\right|_{E}: n \in \mathbb{N}\right\}$ is dense in $C(E, J)$.

To this end, let $g \in C(E, J)$ and $\varepsilon>0$. By Tietze's extension theorem, $g$ can be extended to a function in $C(\mathbb{C})$, also denoted by $g$. We choose $j$ so that $1 / j<\varepsilon / 4$ and $\left\|g-p_{j}\right\|_{E}<\varepsilon / 4$. Since $g(E) \subset J$ we have $\delta\left(p_{j}(E), J\right)<\varepsilon / 4$, and since $E$ belongs to $\mathcal{B}_{j}$ we obtain

$$
\left\|g-f^{\circ N}\right\|_{E} \leq\left\|g-p_{j}\right\|_{E}+\left\|p_{j}-f^{\circ N}\right\|_{E}<\varepsilon / 4+\delta\left(p_{j}(E), J\right)+2 / j<\varepsilon
$$

for some $N \in \mathbb{N}$. 


\section{REFERENCES}

[1] Detlef Bargmann, Simple proofs of some fundamental properties of the Julia set, Ergodic Theory Dynam. Systems 19 (1999), no. 3, 553-558, DOI 10.1017/S0143385799130153. MR.1695942 (2000d:37057)

[2] Walter Bergweiler, Iteration of meromorphic functions, Bull. Amer. Math. Soc. (N.S.) 29 (1993), no. 2, 151-188, DOI 10.1090/S0273-0979-1993-00432-4. MR.1216719 (94c:30033)

[3] Lennart Carleson and Theodore W. Gamelin, Complex dynamics, Universitext: Tracts in Mathematics, Springer-Verlag, New York, 1993. MR1230383 (94h:30033)

[4] Karl-Goswin Grosse-Erdmann, Universal families and hypercyclic operators, Bull. Amer. Math. Soc. (N.S.) 36 (1999), no. 3, 345-381, DOI 10.1090/S0273-0979-99-00788-0. MR:1685272 (2000c:47001)

[5] Alexander S. Kechris, Classical descriptive set theory, Graduate Texts in Mathematics, vol. 156, Springer-Verlag, New York, 1995. MR.1321597(96e:03057)

[6] T. W. Körner, Kahane's Helson curve, Proceedings of the Conference in Honor of Jean-Pierre Kahane (Orsay, 1993), J. Fourier Anal. Appl. 1995, Special Issue, 325-346. MR1364895 (97f:43008)

[7] M. Yu. Lyubich, Generic behavior of trajectories of the exponential function, Uspekhi Mat. Nauk 41 (1986), no. 2(248), 199-200 (Russian). MR842176 (87g:58062)

[8] John Milnor, Dynamics in one complex variable, Friedr. Vieweg \& Sohn, Braunschweig, 1999. Introductory lectures. MR 1721240 (2002i:37057)

[9] Mary Rees, The exponential map is not recurrent, Math. Z. 191 (1986), no. 4, 593-598, DOI 10.1007/BF01162349. MR832817 (87g:58063)

[10] Dierk Schleicher, Dynamics of entire functions, Holomorphic dynamical systems, Lecture Notes in Math., vol. 1998, Springer, Berlin, 2010, pp. 295-339, DOI 10.1007/978-3-642-131714_5. MR2648691 (2011h:37070)

[11] Norbert Steinmetz, Rational iteration: Complex analytic dynamical systems, de Gruyter Studies in Mathematics, vol. 16, Walter de Gruyter \& Co., Berlin, 1993. MR.1224235(94h:30035)

Department of Mathematics, University of Trier, D-54286 Trier, Germany

Department of Mathematics, University of Trier, D-54286 Trier, Germany 